RESEARCH ARTICLE

\title{
Diabetes-specific Modulation of Peripheral Blood Gene Expression Signatures in Colorectal Cancer
}

\author{
Zsuzsanna Molnár $1, \S$, Zsófia Bánlaki ${ }^{1, \S}$, Anikó Somogyi ${ }^{2}$, Zoltán Herold², Magdolna Herold², \\ András Guttman ${ }^{3,4}$, Zsolt Rónai ${ }^{1}$ and Gergely Keszler ${ }^{1, *}$
}

${ }^{1}$ Department of Medical Chemistry, Molecular Biology and Pathobiochemistry, Faculty of Medicine, Semmelweis University, Budapest, Hungary; ${ }^{2}$ 2nd Department of Internal Medicine, Faculty of Medicine, Semmelweis University, Budapest, Hungary; ${ }^{3}$ Horváth Csaba Memorial Laboratory of Bioseparation Sciences, Research Center for Molecular Medicine, Doctoral School of Molecular Medicine, Faculty of Medicine, University of Debrecen, Hungary; ${ }^{4}$ Translational Glycomics Group, Research Institute of Biomolecular and Chemical Engineering, University of Pannonia, Veszprém, Hungary

Abstract: Background: Type 2 diabetes (T2DM) and colorectal cancer (CRC) are both known to modulate gene expression patterns in peripheral blood leukocytes (PBLs).

Objective: As T2DM has been shown to increase the incidence of CRC, we were prompted to check whether diabetes affects mRNA signatures in PBLs isolated from CRC patients.

Methods: Twenty-two patients were recruited to the study and classified into four cohorts (healthy controls; T2DM; CRC; CRC and T2DM). Relative expression levels of

ARTICLE HISTORY

Received: February 14, 2020 Revised: March 27, 2020

Accepted: March 30, 2020

DOI:

10.2174/1566524020666200504084626 573 cell signaling gene transcripts were determined by reverse transcription real-time PCR assays run on low-density OpenArray platforms. Enrichment analysis was performed with the g:GOSt profiling tool to order differentially expressed genes into functional pathways.

Results: 49 genes were found to be significantly up- or downregulated in tumorous diabetic individuals as compared to tumor-free diabetic controls, while 11 transcripts were differentially regulated in patients with CRC versus healthy, tumor-free and nondiabetic controls. Importantly, these gene sets were completely distinct, implying that diabetes exerts a profound influence on the transcription of signaling genes in CRC. The top 5 genes showing the most significant expression differences in both contexts were PCK2, MAPK9, CCND1, HMBS, TLR3 ( $\mathrm{p} \leq 0.0040)$ and CREBBP, PPIA, NFKBIL1, MDM2 and SELPLG $(\mathrm{p} \leq 0.0121)$, respectively. Functional analysis revealed that most significantly affected pathways were cytokine, interleukin and PI3K/Akt/mTOR signaling cascades as well as mitotic regulation.

Conclusion: We propose that differentially expressed genes listed above might be potential biomarkers of CRC and should be studied further on larger patient groups. Diabetes might promote colorectal carcinogenesis by impairing signaling pathways in PBLs.

Keywords: Colorectal carcinoma, Type 2 diabetes, Gene expression, RNA, Blood, Biomarker.

\section{INTRODUCTION}

The past decades have seen rampant increases in the prevalence of both type 2 diabetes (T2DM) and colorectal cancer (CRC) worldwide [1]. It is of note that

\footnotetext{
${ }^{*}$ Address correspondence to this author at the Department of Medical Chemistry, Semmelweis University, POB 2, H-1428 Budapest, Hungary; Tel: +361-4591500; Ext: 60132;

E-mail address: keszler.gergely@med.semmelweis-univ.hu

${ }^{\S}$ These authors contributed equally to this work.
}

both diseases share common risk factors such as obesity, high-sugar and high-fat diet as well as a sedentary lifestyle [2]. Diabetes is a major health burden worldwide as almost $10 \%$ of the human population is affected and the vast majority of them suffer from type 2 diabetes characterized by insulin resistance [3]. While it predisposes for a number of cardiovascular and neurological complications, diabetes has been shown to increase the incidence of several malignancies [4]. A recent meta-analysis of 
numerous epidemiological studies has clearly shown that the incidence of CRC is significantly elevated in general [5] and that of proximal colon tumors in particular [6] in type 2 diabetic individuals. Hyperglycemic patients develop larger primary tumors with lower tumor differentiation [7]. Moreover, type 2 diabetes has been shown to raise the cancer-specific mortality of CRC by $12 \%$ and all-cause mortality of CRC patients by $17 \%$ [8]. Importantly, the risk of CRC seems to be modulated by therapeutic modalities as insulin therapy leads to elevated, while the administration of the pleiotropic metabolic modulator, mTOR inhibitor and AMPK activator metformin, to reduced cancer incidence [9]. These observations raised the possibility that perturbed energy-sensing signaling pathways might underlie diabetes-induced colorectal carcinogenesis. Epigenetic mechanisms have also been suggested [10].

Tumor-promoting molecular pathomechanisms in hyperglycemia and insulin resistance, however, remain largely elusive. The prevailing view suggests that oxidative stress, cytokine imbalance and inflammation elicited by hyperglycemia activate several signaling proteins including MAP kinases and the redox-sensitive transcription factors Nrf2 and NFkB, well-known mediators in malignancies [11]. Hyperinsulinemiainduced mitogenic and antiapoptotic pathways might also stimulate hyperproliferation and malignant transformation of colon epithelial cells [12]. Recent investigations have shed light on the diabetesdependent activation of key mitogenic mechanisms including the $\mathrm{Wnt} / \mathrm{GSK} 3 / \beta$-catenin and epidermal growth factor signaling pathways; well-known players in colorectal carcinogenesis $[13,14]$. Altered microRNA levels have also been suggested to modulate diabetesspecific gene expression changes in cancer cells [15, 16]. Genome-wide association studies have identified a number of genetic polymorphisms as shared risk factors of T2DM and CRC such as the TCF7L2 transcription factor [17], ion channels and transcriptional regulators [18].

While setting up the diagnosis of diabetes by repeatedly checking fasting blood sugar levels or performing a glucose tolerance test is a relatively simple and cheap procedure, it is more problematic to screen large populations by colonoscopy, the current gold standard of CRC diagnosis [19]. Therefore, it would be of great advantage to find easily and quickly measurable, minimally invasive, blood-based detection assays for the early diagnosis of CRC in diabetic individuals. Recent findings imply that soluble mediators released by colorectal cells modulate transcriptomic landscapes in peripheral blood mononuclear cells, providing the rationale for plenty of studies aimed at finding CRC-specific molecular markers - principally altered gene expression patterns - in peripheral blood leukocytes for early diagnosis of CRC in asymptomatic patients [20-22]. Chang et al. performed a global gene expression analysis in PBMC in CRC patients and identified a 5-strong battery of potential biomarker genes (MDM2, DUSP6, CPEB4, $M M D$, and EIF2S3) [20]. Later on, they completed a meta-analysis which revealed that expression patterns of a set of 7 key genes (CPEB4, EIF2S3, MGC20553, MS4A1, ANXA3, TNFAIP6, IL2RB) confirm the diagnosis of CRC with outstanding reliability and specificity [21]. CRC markedly induced the expression of a set of pro-inflammatory proteins (COX-2, TNF, NF$\mathrm{KB}$ and $\mathrm{IL}-6$ ) in human circulating leukocytes in vivo [22]. Direct evidence for the assumption that CRC cells influence leukocyte gene expression patterns via soluble mediators released by tumor cells was provided by Mohebbi et al. [23]. Their results showed that conditioned media collected from cultured Caco-2 human colorectal cancer cells modulated the production of pro- and anti-inflammatory cytokines in vitro in monocytes isolated from normal human blood.

Co-morbidities such as postoperative intraabdominal infections following CRC surgery have been shown to modulate PBL gene expression patterns [24], indicating that special care should be taken when selecting universal CRC-specific gene expression biomarkers.

In light of the rising prevalence of T2DM among patients afflicted by CRC and potential molecular interactions between these devastating diseases, we raised the question whether $\mathrm{CRC}$-associated gene expression signatures of white blood cells were modulated by diabetes as co-morbidity. To address this issue, the expression profiles of a set of signaling genes were analyzed by low-density microarrays in diabetic and non-diabetic CRC patients. Surprisingly, it turned out that diabetes greatly influences these molecular signatures, strongly arguing that the presence of diabetes should be considered in our quest for clinically reliable CRC biomarkers.

\section{MATERIALS AND METHODS}

\subsection{Participants and Sample Collection}

A total of 22 persons were recruited to the present study of which 10 suffered from colorectal adenocarcinoma (CRC), while 12 were tumor-free control individuals. The presence or absence of CRC was verified by colonoscopy in all cases at the $2^{\text {nd }}$ Dept. of Internal Medicine, Semmelweis University, Budapest, Hungary. The diagnosis of CRC was corroborated by tissue sampling and subsequent histopathological examination at the $1^{\text {st }}$ Department of Pathology and Experimental Cancer Research, Semmelweis University, Budapest, Hungary. Control subjects were free from inflammatory bowel disease and intestinal polyposis, whereas CRC subjects were free from any previously diagnosed inflammatory bowel disease and familial adenomatous polyposis syndrome. Individuals with previous malignancies, autoimmune, infectious or metabolic disorders apart from type 2 diabetes (T2DM) as well as patients receiving any kind of anticancer or immunosuppressive therapy were excluded from the study. A summary of exclusion criteria applied for each of the study cohorts is shown in Supplementary Table 1. 
Half of both tumorous and non-tumor individuals ( 5 and 6 persons, respectively) had T2DM. Mean age \pm standard deviation (SD) was $68 \pm 6.53,63 \pm 7.37,63 \pm$ 4.53 and $65 \pm 4.87$ years in the non-tumor + nondiabetic (cohort 1), tumor + non-diabetic (cohort 2), non-tumor + diabetic (cohort 3) and tumor + diabetic (cohort 4), respectively. As far as sex ratios are concerned, cohort 1 comprised 2 males and 4 females, cohort 2 consisted of 3 men and 2 women, cohort 3 contained 3 men and 3 women and the tumor + diabetes group included 2 men and 3 women. The diagnosis of T2DM was set up in line with the latest WHO diagnostic criteria. These patients received proper oral antidiabetic medication (Metformin) and had $\mathrm{HbA}_{1 \mathrm{C}}$ levels below $8 \%$ at the time of CRC diagnosis.

Peripheral blood samples were collected in PAXgene ${ }^{\circledR}$ Blood RNA Tubes (Qiagen, Venlo, The Netherlands) immediately after colonoscopy (the same day) at the $2^{\text {nd }}$ Department of Internal Medicine, Semmelweis University. Participation in the study was voluntary. All participants signed a written informed consent before blood sample collection. This study was approved by the Semmelweis University Regional and Institutional Committee of Science and Research Ethics (permission no. 39/2014) and by the ETTTUKEB (permission No. 8573-9/2017/EÜIG).

\subsection{RNA Extraction and Quantification}

Total RNA was isolated from peripheral blood leukocytes using the MagMAX ${ }^{\mathrm{TM}}$ for Stabilized Blood Tubes RNA Isolation Kit, according to the manufacturer's instructions. Reverse transcription was performed using SuperScript VILO cDNA Synthesis Kit (all reagents were from ThermoFisher Scientific, Waltham, MA). Following cDNA synthesis, equal amounts of samples were pooled in twosomes so that 3 pooled samples per each of the 4 groups were prepared for gene expression analysis (in the case of the five-strong tumor + non-diabetes and the tumor + diabetes groups, two pools and one individual sample were processed).

cDNA levels were quantitated by real-time PCR using the TaqMan ${ }^{\mathrm{TM}}$ OpenArray ${ }^{\mathrm{TM}}$ Human Signal Transduction Panel for QuantStudio ${ }^{\mathrm{TM}} 12 \mathrm{~K}$ Flex (ThermoFisher Scientific). Reactions were performed in triplicate. Expression levels were normalized to the geometrical mean of 24 internal reference genes included on the platform.

\subsection{Statistical and Computer Analyses}

Statistical analyses were carried out by means of the Statistica software version 13.4.0.14 (TIBCO Software Inc, http://tibco.com). Two-tailed unpaired Student's t-test was used for assessing inter-group differences in normalized gene expression values at alpha $=0.05$. Correction for multiple testing was performed by the Benjamini-Hochberg False Discovery Rate (FDR) method.

Enrichment analysis for gene sets showing significant inter-group differences was performed using the g:GOSt functional profiling tool of $\mathrm{g}$ :Profiler (http://biit.cs.ut.ee/gprofiler/) [25] with pathways of the Reactome database (https://reactome.org/) [26] used as a reference.

\section{RESULTS}

In order to analyze the effects of CRC and T2DM alone or in combination on peripheral blood gene expression patterns, four carefully selected, sex and age-matched patient groups were assembled as discovery samples for this pilot study. Total RNA was prepared, reverse transcribed and cDNA levels were quantified using a low-density OpenArray panel that simultaneously interrogated 573 transcripts involved in various signaling pathways. The reason for deploying this microarray was that leukocyte transcriptional signatures are known to be modulated by both malignancies and T2DM [20-22; 27-31], but - to the best of our knowledge - the combined impact of T2DM and CRC on PBL mRNA levels of a large cohort of signaling genes has not been addressed before.

Genes with statistically significant differential expression levels are shown in Suppl. Table 2. Panel A compares gene expression levels in tumorous vs. tumor-free diabetic patients, while the same comparison among non-diabetic individuals is shown in panel B. Finally, panel C displays fold changes irrespective of the diabetes status of CRC patients. Importantly, the majority of differentially expressed genes were downregulated in patients with CRC, and

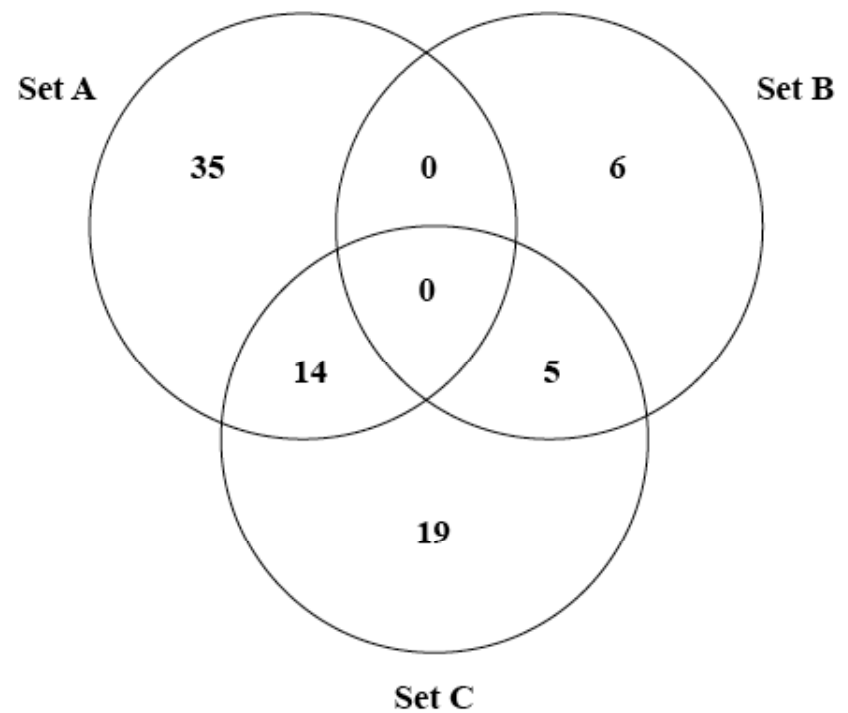

Fig. (1). Overlap of genes showing differential expression.

Graphical representation of data presented in Supplementary Table 1. Sets represent the number of differentially expressed genes in tumor vs. tumor-free samples in diabetic patients (Set A: cohort 3 vs. cohort 4); in non-diabetic subjects (Set B: cohort 1 vs. cohort 2); and in merged groups (Set C: all tumorous compared to all tumor-free individuals, that is, cohorts $2+4$ vs. cohorts $1+3$ ). Numbers in the overlapping segments correspond to genes found to be differentially expressed in more than one sets. 
Table 1. The top 5 transcripts with the most significant expression differences between tumorous and tumor-free individuals in the diabetic (panel A), non-diabetic (B) and merged groups (C).

\begin{tabular}{|c|c|c|c|c|c|c|c|c|}
\hline \multicolumn{3}{|c|}{ Panel A (diabetic group) } & \multicolumn{3}{|c|}{ Panel B (non-diabetic group) } & \multicolumn{3}{|c|}{$\begin{array}{l}\text { Panel C, merged groups (all tumor vs. all } \\
\text { tumor-free patients) }\end{array}$} \\
\hline TaqMan $\circledast$ assay & Gene & $p$ value & TaqMan $\circledast$ assay & Gene & $p$ value & TaqMan $®$ assay & Gene & $p$ value \\
\hline Hs00177102_m1 & MAPK9 & 0.0017 & Hs04194521_s1 & PPIA & 0.0073 & Hs00176481_m1 & $C D K N 2 D$ & 0.0056 \\
\hline Hs00765553_m1 & CCND1 & 0.0024 & Hs00428211_m1 & $N F K B I L 1$ & 0.0084 & Hs00174114_m1 & IL2 & 0.0063 \\
\hline Hs01551078_m1 & $T L R 3$ & 0.0040 & Hs00380945_m1 & SELPLG & 0.0121 & Hs00177957_m1 & MAPKAPKЗ & 0.0089 \\
\hline
\end{tabular}

Statistical significance between mRNA expression in individuals with or without CRC was assessed with a two-tailed unpaired t-test. Transcripts with the most significant expression differences are shown. Genes whose expression was analyzed by two different assays are denoted by an asterisk $\left({ }^{*}\right)$. Transcripts upregulated in tumor vs. tumor-free blood samples are shown in bold, while those downregulated are in italics.

the expression of a few genes was altered more than twofold in any direction. The most striking finding of the study was that there was no overlap at all between the transcriptomic profiles of signaling genes in diabetic and non-diabetic individuals, as illustrated in Fig. 1. (Note that Fig. 1 shows the number of genes but not that of transcripts; that is why there are fewer genes indicated here than transcripts enlisted in Supplementary Table 2). These results argue that the presence or absence of diabetes largely influences the gene expression profiles of peripheral blood leukocytes in CRC patients. In light of these results, the top 5 genes with the most significant expression differences were selected in all three cohorts and displayed in Table 1. These gene cohorts deserve further investigation as potential CRC marker genes in diabetic, non-diabetic or in the general population, respectively.

Finally, a g:GOSt gene enrichment analysis was implemented to identify functionally related gene cohorts among differentially expressed genes, that is, to classify them into functional pathways corresponding to terms in the Reactome database.

Adjusted $p$ values in Table 2 stand for results obtained after multiple testing correction using the g:SCS algorithm optimized specifically for enrichment analyses, taking into account that multiple tests are not independent of each other. Genes associated with a specific term are implicitly associated with parent terms in the terminology hierarchy and functionally annotated gene sets are unevenly distributed. The query gene sets were all the genes showing significant expression difference by t-test between tumor and non-tumor individuals in the diabetic group $(A)$ and the nondiabetic group (B). With regard to the diabetic group, 7 from the total 49 genes could not be included in the analysis (TGFBR3 and TGFBRAP1 were not listed in the database at all, DUSP14 had no match for Homo sapiens, while MAP3K4, TGFBI, MAP4K1 and CREM had matches as "protein" and/ or "interactor" but no matches for "reaction" or "pathway"). In the nondiabetic group, 1 of the total 11 genes found as significant by the t-test was unanalyzable (NFKBIL1 was not found in the database). The wide variety of pathways disturbed in diabetic CRC patients was remarkable as compared to the far fewer number of terms in the non-diabetic cohort. Similarly to the gene sets presented in Fig. 1, the identified terms did not overlap at all between cohorts $A$ and $B$, providing further evidence that diabetes profoundly modulated the signaling pathways in peripheral blood leukocytes of CRC patients.

\section{DISCUSSION}

Recent advances in the field of high-throughput assays made it possible to monitor the entire or partial gene expression signatures in various biological samples [32, 33]. Blood, an easily accessible tissue known to be in contact with all aerobic organs, is an ideal candidate for gene expression studies as several human diseases exert influence on total mRNA landscapes of circulating leukocytes [22, 27, 34]. The aim of the present study was to screen the transcriptome of over half a thousand signaling genes in peripheral blood leukocytes to find easily accessible but reliable diagnostic markers for colorectal carcinoma against the background of type 2 diabetes mellitus, a co-morbidity known to promote cancer development and progression.

Albeit the transcriptomics of both diseases has been studied extensively but separately [20, 31], here we elucidated their combined effect on gene expression using a high-throughput approach. Here, we acknowledge the endeavor of Cui and co-workers [35] who have already conducted a similar study on PBL gene expression in diabetic and CRC individuals, but they focused on five genes only (GRP78, NOX1, CEACAM5, HSP60 and HDAC1) which were not included on our signal transduction platform, making their results not directly comparable to ours. Nevertheless, it is of note that all genes mentioned above showed significantly different expression levels $(p<0.05)$ when tumorous or tumor-free hyperglycemic or normoglycemic patient cohorts were systematically compared.

NFKBIL1, a member of the IKB superfamily, was significantly downregulated in leukocytes isolated from 
Table 2. Reactome database terms with enriched genes showing significant expression differences between tumor and tumor-free individuals.

Panel A

\begin{tabular}{|c|c|c|c|c|c|}
\hline Term & $\begin{array}{l}\text { Adjusted } \\
\mathrm{p} \text { value }\end{array}$ & $\begin{array}{c}\text { Term } \\
\text { size }\end{array}$ & $\begin{array}{l}\text { Query } \\
\text { size }\end{array}$ & $\begin{array}{c}\text { Intersection } \\
\text { size }\end{array}$ & Intersections (genes) \\
\hline Cytokine Signaling in Immune system & 8.73E-08 & 680 & 42 & 17 & $\begin{array}{l}\text { MAPK9, CCND1, BIRC3, CD86, IL2, FGF2, IL12A, BIRC2, CTF1, FASLG, } \\
\text { SOD2, UBE2N, SOS1, CD80, CCL2, EDARADD, PIK3R1 }\end{array}$ \\
\hline Signaling by Interleukins & 4.34E-07 & 461 & 42 & 14 & $\begin{array}{c}\text { MAPK9, CCND1, CD86, IL2, FGF2, IL12A, CTF1, FASLG, SOD2, UBE2N, } \\
\text { SOS1, CD80, CCL2, PIK3R1 }\end{array}$ \\
\hline CD28 dependent $\mathrm{P} \mid 3 \mathrm{~K} / \mathrm{Akt}$ signaling & $6.39 \mathrm{E}-06$ & 22 & 42 & 5 & CD86, AKT3, CD80, PIK3R1, MTOR \\
\hline Signal Transduction & $1.90 \mathrm{E}-04$ & 2670 & 42 & 26 & $\begin{array}{l}\text { CCND1, BIRC3, CD86, PTGDR, IL2, AKT3, BMPR1A, FGF2, GNAS, FZD2, } \\
\text { EPOR, SMAD5, ADRB2, BIRC2, TBP, FASLG, SOS1, CCNE1, CD80, PORCN, } \\
\text { SMAD4, PIK3R1, MTOR, E2F1, DVL2, RASA1 }\end{array}$ \\
\hline $\begin{array}{l}\text { TICAM1, RIP1-mediated IKK complex } \\
\text { recruitment }\end{array}$ & $2.62 \mathrm{E}-04$ & 19 & 42 & 4 & TLR3, BIRC3, BIRC2, UBE2N \\
\hline Costimulation by the CD28 family & $2.05 \mathrm{E}-03$ & 67 & 42 & 5 & CD86, AKT3, CD80, PIK3R1, MTOR \\
\hline Immune System & 2.39E-03 & 2034 & 42 & 21 & $\begin{array}{c}\text { MAPK9, CCND1, TLR3, BIRC3, CD86, MALT1, IL2, AKT3, FGF2, IL12A, } \\
\text { BIRC2, CTF1, FASLG, SOD2, UBE2N, SOS1, CD80, CCL2, EDARADD, } \\
\text { PIK3R1, MTOR }\end{array}$ \\
\hline Mitotic G1-G1/S phases & $7.78 \mathrm{E}-03$ & 148 & 42 & 6 & CCND1, AKT3, PCNA, CDKN2C, CCNE1, E2F1 \\
\hline Disease & 8.63E-03 & 1033 & 42 & 14 & $\begin{array}{l}\text { TLR3, CD86, AKT3, FGF2, TBP, FASLG, SOD2, SOS1, CD80, PORCN, } \\
\text { SMAD4, PIK3R1, MTOR, RASA1 }\end{array}$ \\
\hline Interleukin-10 signaling & $9.35 \mathrm{E}-03$ & 45 & 42 & 4 & CD86, IL12A, CD80, CCL2 \\
\hline Toll Like Receptor 3 (TLR3) Cascade & $9.72 \mathrm{E}-03$ & 92 & 42 & 5 & MAPK9, TLR3, BIRC3, BIRC2, UBE2N \\
\hline Negative regulation of the PI3K/AKT network & 1.13E-02 & 95 & 42 & 5 & CD86, AKT3, FGF2, CD80, PIK3R1 \\
\hline Downstream signaling of activated FGFR3 & 1.77E-02 & 19 & 42 & 3 & FGF2, SOS1, PIK3R1 \\
\hline CTLA4 inhibitory signaling & 2.07E-02 & 20 & 42 & 3 & CD86, AKT3, CD80 \\
\hline IKK complex recruitment mediated by RIP1 & $2.78 \mathrm{E}-02$ & 22 & 42 & 3 & $\mathrm{BIRC} 3, \mathrm{BIRC} 2, \mathrm{UBE} 2 \mathrm{~N}$ \\
\hline $\begin{array}{c}\text { Constitutive Signaling by Aberrant PI3K in } \\
\text { Cancer }\end{array}$ & 3.13E-02 & 61 & 42 & 4 & CD86, FGF2, CD80, PIK3R1 \\
\hline Downstream signaling of activated FGFR4 & 3.19E-02 & 23 & 42 & 3 & FGF2, SOS1, PIK3R1 \\
\hline Signaling by Erythropoietin & 3.64E-02 & 24 & 42 & 3 & EPOR, SOS1, PIK3R1 \\
\hline Downstream signaling of activated FGFR2 & 3.64E-02 & 24 & 42 & 3 & FGF2, SOS1, PIK3R1 \\
\hline Downstream signaling of activated FGFR1 & 3.64E-02 & 24 & 42 & 3 & FGF2, SOS1, PIK3R1 \\
\hline FOXO-mediated transcription & 4.26E-02 & 66 & 42 & 4 & AKT3, FASLG, SOD2, SMAD4 \\
\hline Interleukin receptor SHC signaling & 4.65E-02 & 26 & 42 & 3 & IL2, SOS1, PIK3R1 \\
\hline
\end{tabular}


Panel B

\begin{tabular}{|c|c|c|c|c|c|}
\hline Term & $\begin{array}{c}\text { Adjusted } \\
\text { p value }\end{array}$ & $\begin{array}{c}\text { Term } \\
\text { size }\end{array}$ & $\begin{array}{c}\text { Query } \\
\text { size }\end{array}$ & $\begin{array}{c}\text { Intersection } \\
\text { size }\end{array}$ & CREBBP, BIRC5, CASP1 \\
\hline $\begin{array}{c}\text { TP53 Regulates Transcription of Cell Death } \\
\text { Genes }\end{array}$ & $1.96 \mathrm{E}-03$ & 44 & 10 & 3 & PPIA, MAPKAPK3, BIRC5, RHOU, CASP1 \\
\hline Signaling by Interleukins & $8.11 \mathrm{E}-03$ & 461 & 10 & 5 & CREBBP, MDM2, SRC \\
\hline Transcriptional regulation by RUNX3 & $2.00 \mathrm{E}-02$ & 95 & 10 & 3 & \\
\hline
\end{tabular}

Panel C

\begin{tabular}{|c|c|c|c|c|c|}
\hline & $\begin{array}{c}\text { Adjusted } \\
\mathbf{p} \text { value }\end{array}$ & $\begin{array}{c}\text { Term } \\
\mathbf{s i z e}\end{array}$ & $\begin{array}{c}\text { Query } \\
\mathbf{s i z e}\end{array}$ & $\begin{array}{c}\text { Intersection } \\
\mathbf{s i z e}\end{array}$ & Intersections (genes) \\
\hline Cytokine Signaling in Immune system & $1.16 \mathrm{E}-03$ & 680 & 33 & 11 & CCND1, IL2, MAPKAPK3, SOCS5, IL10RA, TOLLIP, CD80, PPIA, IFNB1, \\
EDARADD, IRAK1
\end{tabular}

Pathways and biological processes identified as significantly altered between tumor and non-tumor individuals by the g:Profiler tool are shown in the diabetic (A), in the non-diabetic (B) and in the merged groups (C). "Term size" stands for the number of genes associated with a specific term in the Reactome database, "Query size" stands for the number of genes analyzed and "Intersection size" indicates the number of matches between the query and a specific term. Transcripts recommended for CRC screening (shown in Table 1) are typed in bold italics.

non-diabetic CRC patients in our study, also implying enhanced NF-KB signaling as suggested by Alamro et al. [22].

Toll-like receptor 3 was found to be significantly downregulated in tumorous diabetic individuals as compared to tumor-free diabetics in our study (Table 1), and some genes from the TLR3 signaling cascade were differentially expressed (Table 2). This finding harmonizes well with the results of Madhumitha and co-workers [36] who observed impaired toll-like receptor signaling in peripheral $B$ lymphocytes of untreated diabetic individuals. Modulation of the Tolllike receptor pathway in CRC was also reported by $\mathrm{Xu}$ et al. [37].

In line with the results of Chang et al. [20], we could also confirm the diagnostic importance of MDM2 as it was significantly altered in our non-diabetic sample, while DUSP14 (but not DUSP6) and IL2 (but not IL2RB) showed differential expression both in the diabetic and total samples. The rest of their diagnostic genes were either unchanged or not included in our signal transduction panel array.

Our results convincingly show that type 2 diabetes mellitus as a comorbidity in CRC dramatically alters signaling activities in PBLs as the spectra of differentially regulated genes were totally different in diabetic vs. non-diabetic CRC subjects (Fig. 1 and Table 2) [24]. The specificity of altered transcriptomic signatures in PBL can be further increased by including a set of genes in the expression analysis and combining transcriptomic data with the determination of serum protein tumor markers such as carcinoembryonic antigen (CEA) and CYFRA21-2 [38].

\section{CONCLUSION}

Taken together, we propose that genes shown in Table 1 (PCK2, MAPK9, CCND1, HMBS, TLR3, CREBBP, PPIA, NFKBIL1, MDM2 and SELPLG), might be potential biomarkers for CRC in diabetic and nondiabetic individuals, underscoring the distinguished role of diabetes in the molecular diagnostics of CRC and should deserve further investigation. CCND1 (cyclin D1) and MAPK9 (JNK) are well-known regulators of cell proliferation, while upregulation of PCK2 (the mitochondrial phosphoenolpyruvate carboxykinase isoform) in diabetic CRC patients might be a common link between diabetes and cancer as it has been shown to promote both gluconeogenesis and tumor formation [39]. In light of the huge impact of diabetes on the expression of a panoply of signaling genes and cascades including cytokine, interleukin and PI3K/ Akt/mTOR signaling as well as cell cycle regulation, our 
results implicate that diabetes-induced inflammation might contribute to the higher incidence and prevalence of $\mathrm{CRC}$ among diabetic individuals.

Please note that in our study we measured mRNA levels of a set of signaling genes only, so it was not a transcriptome-wide global gene expression study. The sample size was rather small, mainly due to the strict inclusion criteria and that we insisted on working with reliable, age-matched groups. This reduced statistical power, hence some genes with small effects are likely to have remained undetected. Finally, it remains elusive whether similar alterations could be observed in mRNA profiles of the tumor tissue per se. To test this issue, we plan to perform the same profiling in surgically removed tumor tissues to see whether gene expression changes observed in $\mathrm{PBL}$ are paralleled by similar alterations in tumor samples too.

\section{ETHICAL APPROVAL AND CONSENT TO PARTICIPATE}

The study was approved by the Semmelweis University Regional and Institutional Committee of Science and Research Ethics (permission no. 39/2014) and by the ETT-TUKEB (permission No. 85739/2017/EÜIG), Budapest, Hungary.

\section{HUMAN AND ANIMAL RIGHTS}

No animals were used in this study. All reported experiments on humans were followed in accordance with the ethical standards of the committee responsible for human experimentation (institutional national), and with the Helsinki Declaration of 1975 , as revised in 2008 (http://www.wma.net/en/20activities/10ethics/ 10helsinki/).

\section{AVAILABILITY OF DATA AND MATERIALS}

All experimental data are shown in the manuscript and supplementary material.

\section{CONSENT FOR PUBLICATION}

Written consent has been obtained from the participants.

\section{FUNDING STATEMENT}

This study was supported by the National Research, Development and Innovation Office (project number: K116128 ) and by the Semmelweis University STIA-KF17 fund.

\section{CONFLICT OF INTEREST}

The authors declare that there is no conflict of interest regarding the publication of this article.

\section{ACKNOWLEDGEMENTS}

None declared.

\section{SUPPLEMENTARY MATERIAL}

Supplementary material is available on the publisher's website along with the published article.

\section{REFERENCES}

[1] GBD 2016 Mortality Collaborators. Global, regional, and national under-5 mortality, adult mortality, age-specific mortality, and life expectancy, 1970-2016: a systematic analysis for the Global Burden of Disease Study. Lancet 2017; 16: 1084-150.

[2] Giouleme O, Diamantidis MD, Katsaros MG. Is diabetes a causal agent for colorectal cancer? Pathophysiological and molecular mechanisms. World J Gastroenterol 2011; 17(4): 444-8.

http://dx.doi.org/10.3748/wjg.v17.i4.444 PMID: 21274373

[3] Ogurtsova K, da Rocha Fernandes JD, Huang Y, et al. IDF Diabetes Atlas: Global estimates for the prevalence of diabetes for 2015 and 2040. Diabetes Res Clin Pract 2017; 128: $40-50$.

http://dx.doi.org/10.1016/j.diabres.2017.03.024

PMID: 28437734

[4] Pan XF, He M, Yu C, et al. China Kadoorie Biobank Collaborative Group. Type 2 Diabetes and Risk of Incident Cancer in China: A Prospective Study Among 0.5 Million Chinese Adults. Am J Epidemiol 2018; 187(7): 1380-91. http://dx.doi.org/10.1093/aje/kwx376 PMID: 29304221

[5] Sun L, Yu S. Diabetes mellitus is an independent risk factor for colorectal cancer. Dig Dis Sci 2012; 57(6): 1586-97. http://dx.doi.org/10.1007/s10620-012-2059-x PMID: 22302244

[6] Demb J, Earles A, Martínez ME, et al. Risk factors for colorectal cancer significantly vary by anatomic site. BMJ Open Gastroenterol 2019; 6(1)e000313

http://dx.doi.org/10.1136/bmjgast-2019-000313 PMID: 31523441

[7] Cui G, Zhang T, Ren F, et al. High blood glucose levels correlate with tumor malignancy in colorectal cancer patients. Med Sci Monit 2015; 21: 3825-33.

http://dx.doi.org/10.12659/MSM.894783 PMID: 26644185

[8] Mills KT, Bellows CF, Hoffman AE, Kelly TN, Gagliardi G. Diabetes mellitus and colorectal cancer prognosis: a metaanalysis. Dis Colon Rectum 2013; 56(11): 1304-19. http://dx.doi.org/10.1097/DCR.0b013e3182a479f9 24105007

[9] Dulskas A, Patasius A, Linkeviciute-Ulinskiene D, Zabuliene L, Urbonas V, Smailyte G. Metformin increases cancer specific survival in colorectal cancer patients-National cohort study. Cancer Epidemiol 2019; 62101587 http://dx.doi.org/10.1016/j.canep.2019.101587

PMID: 31491730

[10] Yang J, Nishihara R, Zhang X, Ogino S, Qian ZR. Energy sensing pathways: Bridging type 2 diabetes and colorectal cancer? J Diabetes Complications 2017; 31(7): 1228-36. http://dx.doi.org/10.1016/j.jdiacomp.2017.04.012 PMID: 28465145

[11] Arcambal A, Taïlé J, Rondeau P, Viranaïcken W, Meilhac O, Gonthier MP. Hyperglycemia modulates redox, inflammatory and vasoactive markers through specific signaling pathways in cerebral endothelial cells: Insights on insulin protective action. Free Radic Biol Med 2019; 130: 59-70.

http://dx.doi.org/10.1016/j.freeradbiomed.2018.10.430 PMID: 30359759

[12] Gunter MJ, Hoover DR, Yu H, et al. Insulin, insulin-like growth factor-I, endogenous estradiol, and risk of colorectal cancer in postmenopausal women. Cancer Res 2008; 68(1): 329-37. http://dx.doi.org/10.1158/0008-5472.CAN-07-2946 PMID: 18172327

Ivonne Wence-Chavez L, Palomares-Chacon U, Pablo Flores-Gutierrez J, et al. Gene expression profiling demonstrates WNT/ $\beta$-catenin pathway genes alteration in Mexican patients with colorectal cancer and diabetes mellitus. J BUON 2017; 22(5): 1107-14. 
PMID: 29135090

[14] Jiao L, Chen L, Alsarraj A, Ramsey D, Duan Z, El-Serag HB. Plasma soluble receptor for advanced glycation end-products and risk of colorectal adenoma. Int $\mathrm{J}$ Mol Epidemiol Genet 2012; 3(4): 294-304

PMID: 23205181

[15] Cirillo F, Catellani C, Sartori C, Lazzeroni P, Amarri S, Street ME. Obesity, Insulin Resistance, and Colorectal Cancer: Could miRNA Dysregulation Play A Role? Int J Mol Sci 2019; 20(12): 12. http://dx.doi.org/10.3390/ijms20122922 PMID: 31207998

[16] Yang IP, Tsai HL, Huang CW, et al. High blood sugar levels significantly impact the prognosis of colorectal cancer patients through down-regulation of microRNA-16 by targeting Myb and VEGFR2. Oncotarget 2016; 7(14): 1883750 .

http://dx.doi.org/10.18632/oncotarget.7719 PMID: 26934556

[17] Sainz J, Rudolph A, Hoffmeister M, et al. Effect of type 2 diabetes predisposing genetic variants on colorectal cancer risk. J Clin Endocrinol Metab 2012; 97(5): E845-51. http://dx.doi.org/10.1210/jc.2011-2565 PMID: 22419714

[18] Cheng I, Caberto CP, Lum-Jones A, et al. Type 2 diabetes risk variants and colorectal cancer risk: the Multiethnic Cohort and PAGE studies. Gut 2011; 60(12): 1703-11. http://dx.doi.org/10.1136/gut.2011.237727 PMID: 21602532

[19] Gkolfakis P, Tziatzios G, Dimitriadis GD, Triantafyllou K. New endoscopes and add-on devices to improve colonoscopy performance. World J Gastroenterol 2017; 23(21): 3784-96. http://dx.doi.org/10.3748/wjg.v23.i21.3784 PMID: 28638218

[20] Chang YT, Huang CS, Yao CT, et al. Gene expression profile of peripheral blood in colorectal cancer. World J Gastroenterol 2014; 20(39): 14463-71.

http://dx.doi.org/10.3748/wjg.v20.i39.14463 PMID: 25339833

[21] Chang YT, Yao CT, Su SL, et al. Verification of gene expression profiles for colorectal cancer using 12 internet public microarray datasets. World J Gastroenterol 2014; 20(46): 17476-82.

http://dx.doi.org/10.3748/wjg.v20.i46.17476 PMID: 25516661

[22] Alamro RA, Mustafa M, Al-Asmari AK. Inflammatory gene mRNA expression in human peripheral blood and its association with colorectal cancer. J Inflamm Res 2018; 11: 351-7.

http://dx.doi.org/10.2147/JIR.S155507 PMID: 30288078

[23] Mohebbi B, Ashtibaghaei K, Hashemi M, Hashemi M, Asadzadeh Aghdaei H, Zali MR. Conditioned Medium from Cultured Colorectal Cancer Cells Affects Peripheral Blood Mononuclear Cells Inflammatory Phenotype in Vitro. Iran J Med Sci 2019; 44(4): 334-41.

PMID: 31439977

[24] Alonso S, Mayol X, Nonell L, Salvans S, Pascual M, Pera M. Colorectal Cancer Research Group. (Hospital del Mar Medical Research Institute). Peripheral blood leucocytes show differential expression of tumour progression-related genes in colorectal cancer patients who have a postoperative intra-abdominal infection: a prospective matched cohort study. Colorectal Dis 2017; 19: 115-25.

http://dx.doi.org/10.1111/codi.13635

[25] Reimand J, Arak T, Adler P, et al. g:Profiler-a web server for functional interpretation of gene lists (2016 update). Nucleic Acids Res 2016; 44(W1): W83-9.

http://dx.doi.org/10.1093/nar/gkw199 PMID: 27098042

[26] Fabregat A, Jupe S, Matthews L, et al. The Reactome Pathway Knowledgebase. Nucleic Acids Res 2018; 46(D1): D649-55.

http://dx.doi.org/10.1093/nar/gkx1132 PMID: 29145629

[27] Gao L, Sun N, Xu Q, Jiang Z, Li C. Comparative analysis of mRNA expression profiles in Type 1 and Type 2 diabetes mellitus. Epigenomics 2019; 11(6): 685-99. http://dx.doi.org/10.2217/epi-2018-0055 PMID: 31016992
[28] Takamura T, Honda M, Sakai $Y$, et al. Gene expression profiles in peripheral blood mononuclear cells reflect the pathophysiology of type 2 diabetes. Biochem Biophys Res Commun 2007; 361(2): 379-84.

http://dx.doi.org/10.1016/j.bbrc.2007.07.006 PMID: 17651698

[29] Grayson BL, Wang L, Aune TM. Peripheral blood gene expression profiles in metabolic syndrome, coronary artery disease and type 2 diabetes. Genes Immun 2011; 12(5): 34151.

http://dx.doi.org/10.1038/gene.2011.13 PMID: 21368773

[30] Stoynev N, Dimova I, Rukova B, et al. Gene expression in peripheral blood of patients with hypertension and patients with type 2 diabetes. J Cardiovasc Med (Hagerstown) 2014; 15(9): 702-9.

http://dx.doi.org/10.2459/JCM.0b013e32835dbcc8 PMID: 23337395

[31] Manoel-Caetano FS, Xavier DJ, Evangelista AF, et al. Gene expression profiles displayed by peripheral blood mononuclear cells from patients with type 2 diabetes mellitus focusing on biological processes implicated on the pathogenesis of the disease. Gene 2012; 511(2): 151-60. http://dx.doi.org/10.1016/j.gene.2012.09.090

PMID: 23036710

[32] Sun MA, Shao X, Wang Y. Microarray Data Analysis for Transcriptome Profiling. Methods Mol Biol 2018; 1751: 17-33. http://dx.doi.org/10.1007/978-1-4939-7710-9_2 PMID: 29508287

[33] Patel SN, Wu Y, Bao Y, Mancebo R, Au-Young J, Grigorenko E. TaqMan ${ }^{\circledR}$ OpenArray ${ }^{\circledR}$ high-throughput transcriptional analysis of human embryonic and induced pluripotent stem cells. Methods Mol Biol 2013; 997: 191-201.

http://dx.doi.org/10.1007/978-1-62703-348-0_15 PMID: 23546757

[34] Dang X, Qu X, Wang W, et al. Bioinformatic analysis of microRNA and mRNA Regulation in peripheral blood mononuclear cells of patients with chronic obstructive pulmonary disease. Respir Res 2017; 18(1): 4. http://dx.doi.org/10.1186/s12931-016-0486-5

PMID: 28057018

[35] Cui G, Feng WM, Zhang T, et al. [Validation and Evaluation of Diagnostic Efficiency of Genes Associated with Colorecta Cancer with Hyperglycemia]. Zhongguo Yi Xue Ke Xue Yuan Xue Bao 2018; 40(6): 769-77.

PMID: 30606387

[36] Madhumitha H, Mohan V, Kumar NP, Pradeepa R, Babu S, Aravindhan V. Impaired toll-like receptor signalling in peripheral B cells from newly diagnosed type-2 diabetic subjects. Cytokine 2015; 76(2): 253-9.

http://dx.doi.org/10.1016/j.cyto.2015.04.010 PMID: 25959254

[37] $\mathrm{Xu} Y, \mathrm{Xu} Q$, Yang L, et al. Gene expression analysis of peripheral blood cells reveals Toll-like receptor pathway deregulation in colorectal cancer. PLoS One 2013; $8(5) \mathrm{e} 62870$

http://dx.doi.org/10.1371/journal.pone. 0062870

PMID: 23650534

[38] Ciarloni L, Ehrensberger SH, Imaizumi N, et al. Development and Clinical Validation of a Blood Test Based on 29-Gene Expression for Early Detection of Colorectal Cancer. Clin Cancer Res 2016; 22(18): 4604-11.

http://dx.doi.org/10.1158/1078-0432.CCR-15-2057 PMID: 27126992

[39] Méndez-Lucas A, Hyroššová P, Novellasdemunt L, Viñals F, Perales JC. Mitochondrial phosphoenolpyruvate carboxykinase (PEPCK-M) is a pro-survival, endoplasmic reticulum (ER) stress response gene involved in tumor cell adaptation to nutrient availability. J Biol Chem 2014; 289(32): 22090-102.

http://dx.doi.org/10.1074/jbc.M114.566927 PMID: 24973213 